\title{
Spinal cord injury and quality of life: a systematic review of outcome measures
}

\author{
Authors Jefferson R Wilson', Robin E Hashimoto², Joseph R Dettori², Michael G Fehlings ${ }^{1}$ \\ Institutions 1 University of Toronto, Toronto Western Hospital, Ontario, Canada \\ 2 Spectrum Research Inc, Tacoma, WA, USA
}

\section{ABSTRACT}

Study design: Systematic review.

Study rationale: According to current estimates, there are more than 1 million people living with a spinal cord injury (SCI) in the United States alone. Given the potentially devastating impact of SCI on health-related quality of life (QoL), we sought to gain an improved understanding of QoL outcomes in SCI.

Objective: To identify and describe common QoL outcomes measures in patients with SCI.

Methods: A systematic review of the English-language literature was undertaken for articles published from 1998 through December 2010. Electronic databases and reference lists of key articles were searched to identify measures or indices used to evaluate QoL outcomes in patients with SCI. The titles and abstracts of the SCI peer-reviewed literature were searched to determine which of these outcome measures were most commonly used to evaluate QoL in patients with SCI.

Results: We identified 27 outcome measures used to evaluate QoL in patients with SCI. In SCI literature, the six most commonly used objective outcome measures were the Short-form 36 (SF-36); Craig Handicap Assessment and Reporting Technique (CHART); Short-form 12 (SF-12); Sickness Impact Profile (SIP68); Reintegration to Normal Living Index (RNL); and Community Integration Questionnaire (CIQ). The six subjective measures that were most frequently used were the Satisfaction with Life Scale (SWLS); Quality of Life Index (QLI); Life Satisfaction Questionnaire (LISAT-9/-1); World Health Organization Quality of Life-BREF scale (WHOQOL-BREF); Perceived Quality of Life (PQOL); and global QoL. All six objective measures have been validated in an SCI population, and four of the six subjective measures have been similarly validated. Three of each of the objective and subjective measures have been reliability tested in a population with SCI.

Conclusion: In addition to neurological and functional changes after SCI, QoL outcomes should be routinely assessed. Choice of appropriate QoL measure should be influenced by the study objectives and design, as well as the psychometric properties of the particular measure within the context of SCI.

This systematic review was funded by AOSpine 


\section{STUDY RATIONALE AND CONTEXT}

The World Health Organization's International Classification of Functioning, Disability and Health encourages the incorporation of a comprehensive definition of health when examining patient-related outcomes after injury or disease [1]. Accordingly, in evaluating recovery after spinal cord injury (SCI), there is increasing focus on evaluating patients' changes in overall well-being, or quality of life (QoL), in addition to neurological and functional changes over time. To date, a QoL outcome assessment tool specific for and validated on patients with SCI remains elusive. One of the greatest challenges in achieving this goal remains creating a tool sensitive to the varied clinical spectrum of SCI, since the definition of QoL may vary dramatically depending on the level and severity of injury. Although we lack an SCI-specific QoL measurement tool, numerous QoL outcome measures intended for use on generic or disease-specific patient populations have been used in studies examining recovery after SCI.

\section{OBJECTIVES}

To identify and describe QoL outcome measures commonly used to assess patients with SCI and to evaluate which of these tools is most appropriate for the SCI population.

\section{MATERIALS AND METHODS}

Study design: Systematic review.

\section{Sampling:}

- Search: PubMed; bibliographies of key articles.

- Dates searched: 1998 through December 2010.

Inclusion criteria: (1) spinal cord injury (SCI); (2) adults 18 years and older; (3) studies reporting on QoL outcome measures.

Exclusion criteria: (1) cancer; (2) infection; (3) children or adolescents.

Outcomes: QoL stratified as "objective quality of life" or "subjective quality of life"[2]. Objective QoL refers to fulfilling the cultural and societal definitions of material wealth, social status, and physical well-being. Subjective QoL considers individuals‘ assessment of their emotions, happiness, or satisfaction with respect to their expectations and achievements.

Analysis: identification of QoL outcome measures commonly reported in studies of patients with SCI and estimation of the frequency of their use in the SCI literature.

Details about methods can be found in the web appendix at www.aospine.org/ebsj.

\section{RESULTS}

We identified 27 QoL outcome measures used in the SCI literature that met our inclusion criteria (Fig 1). Fifteen of these measurement tools may be considered objective, while twelve are subjective in nature. We determined the frequency of use of each measure in the SCI literature (1998 through December 2010) as reported in the abstract: the six most commonly reported objective and subjective measures are further described. 


\section{Objective outcome measures used to evaluate QoL in patients with SCI (Fig 1 and Table 1)}

- Fifteen objective outcome measures were identified.

- The six most commonly used objective outcome measures were: Short-form 36 (SF-36); Craig Handicap Assessment and Reporting Technique (CHART); Short-form 12 (SF-12); Sickness Impact Profile (SIP68); Reintegration to Normal Living Index (RNL); and Community Integration Questionnaire (CIQ). A summary of each outcome measure is available in Table $\mathbf{1 .}$

- Of these,

- None were developed in an SCI population: three are specific to patients with disabilities and/or injuries (CIQ, CRAIG, and RNL), while three were developed for the generic patient population.

- All have been validated in an SCI population.

- Three have been tested for reliability in an SCI population.

- These six objective measures were developed to evaluate the following outcomes: community integration following traumatic brain injury (CIQ) [3, 4]; level of handicap (CHART)[5, 6]; satisfaction with activities of daily living following an incapacitating illness or trauma (RNL) [7, 8]; health-related changes in behavior associated with the activities of daily living (SIP68) [9]; and general function and well-being (SF-36 and SF-12) [10, 11].

\section{Subjective outcome measures used to evaluate QoL in patients with SCI (Fig 1 and Table 2)}

- Twelve subjective outcome measures were identified.

- The six most commonly used objective outcome measures were: Satisfaction with Life Scale (SWLS); Quality of Life Index (QLI); Life Satisfaction Questionnaire LISAT-9/-1); World Health Organization Quality of LifeBREF scale (WHOQOL-BREF); Perceived Quality of Life (PQOL); and global QoL. The scale and interpretation of each is summarized in Table $\mathbf{1}$.

Of these,

- All were developed in a generic popu-

lation, though an SCI-specific ver-

sion of the QLI is available.

- Four have been validated in an SCI population.

- Three have been tested for reli-

ability in an SCI population.

- These six subjective outcome measures were designed to assess the following: life satisfaction in various life domains (LISAT-9/-1 [12, 13], PQOL [14], QLI [14], and WHOQOL-BREF $[15,16])$; and overall perception of QoL or life satisfaction (SWLS [17]; global QoL scale).
Fig 1 Common measures used to evaluate quality of life (QoL) outcomes in patients with spinal cord injury (SCl): number of SCl studies* using each measuret.

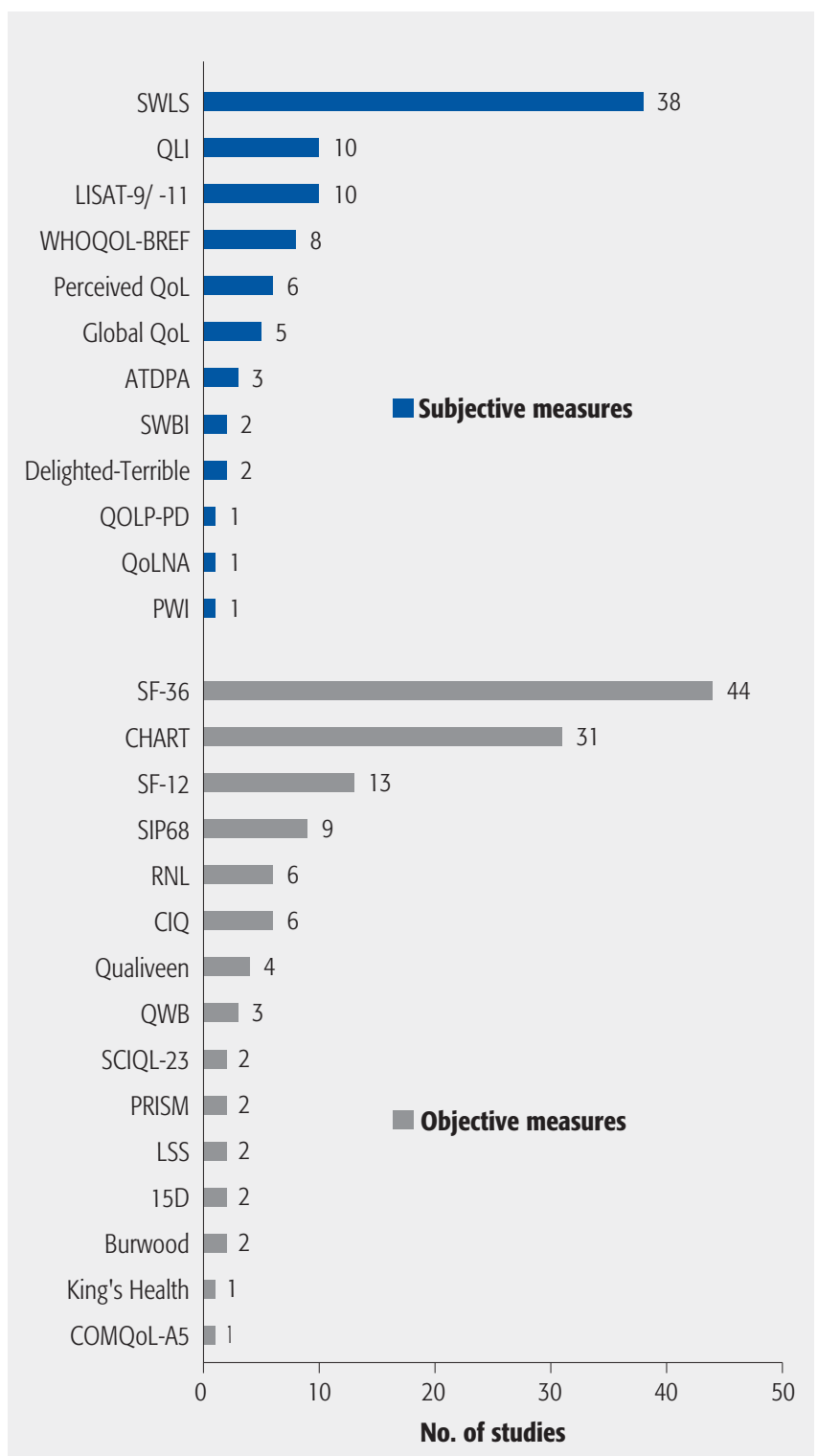

SWLS indicates Satisfaction with Life Scale; QLI, Quality of Life Index; LISAT-9/-11, Life Satisfaction Questionnaire; WHOQOL-BREF, World Health Organization Quality of Life-BREF scale; ATDPA, Assistive Technology Device Predisposition Assessment; SWBI, Sense of Well-being Index; QOLP-PD, Quality of Life Profile for Adults with Physical Disabilities; QOLNA, Quality of Life \& Needs Assessment; PWI, Personal Well-Being Index; SF-36, Short form-36; CHART, Craig Handicap Assessment and Reporting Technique; SF-12, Short-form-12; SIP68, Sickness Impact Profile; RNL, Reintegration to Normal Living Index; CIQ, Community Integration Questionnaire; QWB, Quality of Well-Being Scale; SIQL-23, Spinal Cord Injuries Quality of Life-23-item questionnaire; PRISM, Patient Reported Impact of Spasticity Measure; LSS, Leisure Satisfaction Scale; 15D, Fifteen-dimensional self-administered instrument; and COMQOL-A5, Comprehensive Quality of Life Scale for Adults v. 5

* Citations from PubMed from 1998 through December 2010.

${ }^{\dagger}$ As reported in the title and abstract. 
Table 1 Objective quality of life (QoL) outcomes measures used in assessing patients with spinal cord injury $(\mathrm{SCl})$.

\begin{tabular}{|c|c|c|c|c|c|}
\hline $\begin{array}{l}\text { Instru- } \\
\text { ment }\end{array}$ & $\begin{array}{l}\text { Spine- } \\
\text { specific? }\end{array}$ & Scale & Interpretation & $\begin{array}{l}\text { Validated in } \\
\text { SCI } \\
\text { population? }\end{array}$ & $\begin{array}{l}\text { Reliability } \\
\text { tested in SCI } \\
\text { population? }\end{array}$ \\
\hline \multirow{3}{*}{ 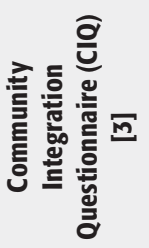 } & \multirow{3}{*}{$\begin{array}{l}\text { No } \\
\text { (traumatic } \\
\text { brain } \\
\text { injury) }\end{array}$} & \multirow{3}{*}{$\begin{array}{l}15 \text { items relating to following subscales: } \\
\text { - Home integration (5 items) } \\
\text { - Social integration (5 items) } \\
\text { - Productive activities (5 items) }\end{array}$} & $\begin{array}{l}\text { Overall score is sum of scores of the individual } \\
\text { scores; subscores may also be calculated [4] }\end{array}$ & \multirow[t]{3}{*}{ Yes [21] } & \multirow[t]{3}{*}{ No } \\
\hline & & & $\begin{array}{l}\text { Maximum subscale score: } 29 \\
\text { Minimum subscale score: } 0\end{array}$ & & \\
\hline & & & $\begin{array}{l}\text { Higher the score, greater is community } \\
\text { integration }\end{array}$ & & \\
\hline \multirow{4}{*}{ 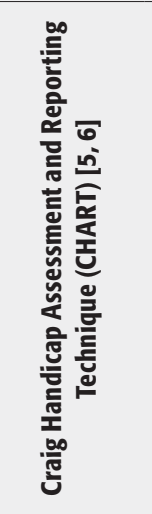 } & \multirow{4}{*}{$\begin{array}{l}\text { No } \\
\text { (disability } \\
\text { related) }\end{array}$} & \multirow{4}{*}{$\begin{array}{l}\text { 32/19 items (long/short form) relating to the } \\
\text { following subscales: } \\
\text { - Physical independence ( } 3 / 1 \text { items: long/short form) } \\
\text { - Cognitive independence ( } 5 / 2 \text { items: long/short } \\
\text { form) } \\
\text { - Mobility ( } 9 / 3 \text { items: long/short form) } \\
\text { - Occupation ( } 7 / 5 \text { items: long/short form) } \\
\text { - Social integration ( } 6 / 6 \text { items: long/short form) } \\
\text { - Economic self-sufficiency ( } 2 / 2 \text { items: long/short } \\
\text { form) } \\
\text { Reported health transition ( } 1 \text { item) is used to } \\
\text { measure changes in health status. It is not included in } \\
\text { any of the subscales and is administered as a } \\
\text { supplemental question }\end{array}$} & $\begin{array}{l}\text { Dimension scores and total CHART scores can } \\
\text { be calculated using the scoring form [6] }\end{array}$ & \multirow[t]{4}{*}{ Yes [21-25] } & \multirow[t]{4}{*}{ Yes [23-25] } \\
\hline & & & $\begin{array}{l}\text { Maximum subscale score: } 100 \\
\text { Minimum subscale score: } 0\end{array}$ & & \\
\hline & & & \multirow[t]{2}{*}{ Lower the score, greater is the handicap } & & \\
\hline & & & & & \\
\hline \multirow{3}{*}{ 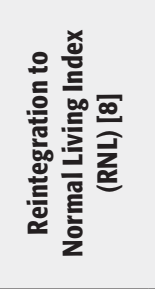 } & \multirow{3}{*}{$\begin{array}{l}\text { No } \\
\text { (incapaci- } \\
\text { tating } \\
\text { illness or } \\
\text { trauma } \\
\text { related) }\end{array}$} & \multirow{3}{*}{$\begin{array}{l}11 \text { items each graded using 11-point visual-analogue } \\
\text { scale (VAS) [8]: } \\
\text { - Mobility (3 items) } \\
\text { - Self-care (1 item) } \\
\text { - Vocation (1 item) } \\
\text { - Recreational/leisure activities ( } 1 \text { item) } \\
\text { - Family/social relationships (4 items) } \\
\text { - Coping ability (1 item) }\end{array}$} & $\begin{array}{l}\text { Total (sum) and adjusted }((\text { total } \times 100) / 110) \\
\text { scores can be calculated [7] }\end{array}$ & \multirow[t]{3}{*}{ Yes [26] } & \multirow[t]{3}{*}{ No } \\
\hline & & & Maximum total/adjusted score: 110/100 & & \\
\hline & & & $\begin{array}{l}\text { Minimum total/adjusted score: 0/0 } \\
\text { Higher the score, the more complete the } \\
\text { reintegration }\end{array}$ & & \\
\hline \multirow{5}{*}{ 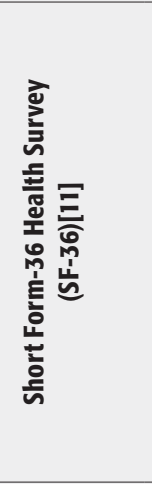 } & \multirow[t]{5}{*}{$\begin{array}{l}\text { No } \\
\text { (generic) }\end{array}$} & \multirow{5}{*}{$\begin{array}{l}36 \text { items relating to following subscales: } \\
\text { - Physical functioning ( } 10 \text { items) } \\
\text { - Physical role limitations ( } 4 \text { items) } \\
\text { - Bodily pain ( } 2 \text { items) } \\
\text { - General health ( } 5 \text { items) } \\
\text { - Vitality (4 items) } \\
\text { - Social functioning ( } 2 \text { items) } \\
\text { - Emotional role limitations ( } 3 \text { items) } \\
\text { - Mental health (5 items) } \\
\text { Reported health transition ( } 1 \text { item) is used to } \\
\text { measure changes in health status. It is not included } \\
\text { in any of the subscales and is administered as a } \\
\text { supplemental question }\end{array}$} & $\begin{array}{l}\text { Items of each subscale are averaged to } \\
\text { yield a score of } 0-100\end{array}$ & \multirow[t]{5}{*}{ Yes [27-30] } & \multirow[t]{5}{*}{ Yes $[27,29,30]$} \\
\hline & & & $\begin{array}{l}\text { Maximum subscale score: } 100 \\
\text { Minimum subscale score: } 0\end{array}$ & & \\
\hline & & & Lower the score, greater the disability & & \\
\hline & & & \multirow{2}{*}{$\begin{array}{l}\text { Norm-based scoring (NBS) can be used to } \\
\text { score all } 3 \text { SF surveys. Through NBS, scale and } \\
\text { summary scores are standardized to a mean of } \\
50 \text { and a standard deviation of } 10 \text { in general US } \\
\text { population, allowing scores to be compared } \\
\text { within and across different SF surveys }\end{array}$} & & \\
\hline & & & & & \\
\hline \multirow{7}{*}{ 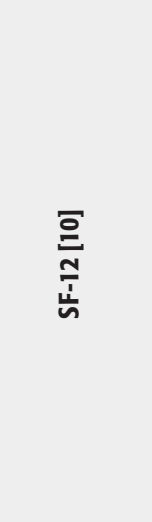 } & \multirow[t]{7}{*}{$\begin{array}{l}\text { No } \\
\text { (generic) }\end{array}$} & \multirow{6}{*}{$\begin{array}{l}\text { Abbreviated version of SF-36: } 12 \text { items relating to } \\
\text { following } 2 \text { domains: } \\
\text { Physical health } \\
\text { - Physical functioning ( } 2 \text { items) } \\
\text { - Physical role limitations ( } 2 \text { items) } \\
\text { - Bodily pain ( } 1 \text { item) } \\
\text { - General health ( } 1 \text { item) } \\
\text { Mental health } \\
\text { - Vitality/mental health ( } 3 \text { items) } \\
\text { - Social functioning ( } 1 \text { item) } \\
\text { - Emotional role limitations ( } 2 \text { items) }\end{array}$} & $\begin{array}{l}\text { Sum items for each domain to yield a physical } \\
\text { health score and mental health score }\end{array}$ & \multirow[t]{7}{*}{ Yes $[22,28,31]$} & \multirow[t]{7}{*}{ No } \\
\hline & & & Sum 2 domains for total score & & \\
\hline & & & $\begin{array}{l}\text { Maximum total score: } 47 \\
\text { Minimum total score: } 12\end{array}$ & & \\
\hline & & & $\begin{array}{l}\text { Maximum physical health score: } 20 \\
\text { Minimum physical health score: } 6\end{array}$ & & \\
\hline & & & $\begin{array}{l}\text { Maximum mental health score: } 27 \\
\text { Minimum mental health score: } 6\end{array}$ & & \\
\hline & & & \multirow[t]{2}{*}{ Lower the score, greater the disability } & & \\
\hline & & Each item scored on variable 1- to 6-point scale & & & \\
\hline
\end{tabular}


Table 1 (Cont.)

\begin{tabular}{|c|c|c|c|c|c|}
\hline $\begin{array}{l}\text { Instru- } \\
\text { ment }\end{array}$ & $\begin{array}{l}\text { Spine- } \\
\text { specific? }\end{array}$ & Scale & Interpretation & $\begin{array}{l}\text { Validated in } \\
\mathrm{SCI} \\
\text { population? }\end{array}$ & $\begin{array}{l}\text { Reliability } \\
\text { tested in SCI } \\
\text { population? }\end{array}$ \\
\hline \multirow{8}{*}{ 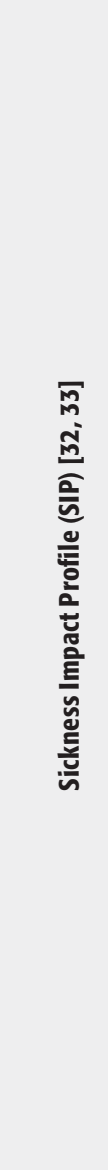 } & \multirow[t]{8}{*}{$\begin{array}{l}\text { No } \\
\text { (generic) }\end{array}$} & \multirow{4}{*}{$\begin{array}{l}136 \text { items relating to following categories: } \\
\text { - Sleep and rest ( } 7 \text { items) } \\
\text { - Eating ( } 9 \text { items) } \\
\text { - Work ( } 9 \text { items) } \\
\text { - Home management (10 items) } \\
\text { - Recreation ( } 8 \text { items) } \\
\text { - Ambulation ( } 12 \text { items) } \\
\text { - Mobility (10 items) } \\
\text { - Self-care ( } 23 \text { items) } \\
\text { - Social interaction ( } 20 \text { items) } \\
\text { - Alertness ( } 10 \text { items) } \\
\text { - Emotional behavior ( } 9 \text { items) } \\
\text { - Communication ( } 9 \text { items) }\end{array}$} & $\begin{array}{l}\text { For each category, dimension and overall } \\
\text { score, scores are summed and expressed as } \\
\text { percentage of maximum score possible }\end{array}$ & \multirow[t]{8}{*}{ Yes $[9,34]$} & \multirow[t]{8}{*}{ Yes $[9,34]$} \\
\hline & & & $\begin{array}{l}\text { Scores can be calculated for each category: } \\
\text { physical dimension, psychosocial dimension, } \\
\text { and overall }\end{array}$ & & \\
\hline & & & $\begin{array}{l}\text { Maximum category, dimension, and overall } \\
\text { score: } 100 \\
\text { Minimum category, dimension, and overall } \\
\text { score: } 0\end{array}$ & & \\
\hline & & & \multirow[t]{5}{*}{ Higher the score, greater the disability } & & \\
\hline & & $\begin{array}{l}\text { The following can be further aggregated into a } \\
\text { physical dimension: } \\
\text { - Ambulation } \\
\text { - Mobility } \\
\text { - Self-care }\end{array}$ & & & \\
\hline & & $\begin{array}{l}\text { The following can be further aggregated into a } \\
\text { psychosocial dimension: } \\
\text { - Social interaction } \\
\text { - Alertness } \\
\text { - Emotional behavior } \\
\text { - Communication }\end{array}$ & & & \\
\hline & & $\begin{array}{l}\text { The following are independent and each can be } \\
\text { scored separately: } \\
\text { - Sleep and rest } \\
\text { - Eating } \\
\text { - Work } \\
\text { - Home management }\end{array}$ & & & \\
\hline & & $\begin{array}{l}\text { Each item is a "yes" ( } 1 \text { point) or "no" (0 point) } \\
\text { statement }\end{array}$ & & & \\
\hline
\end{tabular}


Table 2 Subjective quality of life (QoL) outcomes measures used in assessing patients with spinal cord injury (SCl).

\begin{tabular}{|c|c|c|c|c|c|}
\hline $\begin{array}{l}\text { Instru- } \\
\text { ment }\end{array}$ & $\begin{array}{l}\text { Generic } \\
\text { or spine } \\
\text { specific? }\end{array}$ & Scale & Interpretation & $\begin{array}{l}\text { Validated in } \\
\mathrm{SCI} \\
\text { population? }\end{array}$ & $\begin{array}{l}\text { Reliability } \\
\text { tested in SCI } \\
\text { population? }\end{array}$ \\
\hline \multirow{2}{*}{ 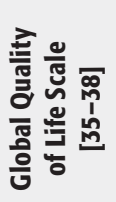 } & \multirow[t]{2}{*}{$\begin{array}{l}\text { No } \\
\text { (generic) }\end{array}$} & \multirow[t]{2}{*}{$\begin{array}{l}\text { Visual analogue scale }(0-100 \mathrm{~mm}) \text { [35-38] based on } \\
\text { the pain VAS [39] }\end{array}$} & $\begin{array}{l}\text { Minimum score: } 0 \\
\text { Maximum score: } 100 \text { mm }\end{array}$ & \multirow[t]{2}{*}{ No } & \multirow[t]{2}{*}{ No } \\
\hline & & & Higher the score, greater the QoL [35-39] & & \\
\hline \multirow{3}{*}{ 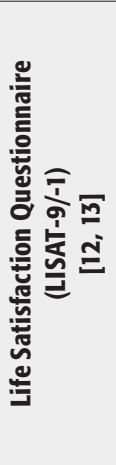 } & \multirow[t]{3}{*}{$\begin{array}{l}\text { No } \\
\text { (generic) }\end{array}$} & \multirow{3}{*}{$\begin{array}{l}9 \text { (or 11) items each graded using 6-point Likert scale } \\
{[12,13,40] \text { : }} \\
\text { General life satisfaction } \\
\text { - Self-care } \\
\text { - Vocational } \\
\text { - Financial } \\
\text { - Leisure situation } \\
\text { - Sexual life } \\
\text { Partner relationship } \\
\text { - Family life } \\
\text { - Social contacts } \\
\text { - Somatic health (LISAT-11 only) } \\
\text { - Psychological health (LISAT-11 only) }\end{array}$} & $\begin{array}{l}\text { Scores derived by taking mean of sum scores } \\
\text { for all items [40] }\end{array}$ & \multirow[t]{3}{*}{ Yes $[22,34,41]$} & \multirow[t]{3}{*}{ Yes $[34,41]$} \\
\hline & & & $\begin{array}{l}\text { Minimum score: } 1 \\
\text { Maximum score: } 6\end{array}$ & & \\
\hline & & & Higher the score, greater the satisfaction & & \\
\hline \multirow{4}{*}{ 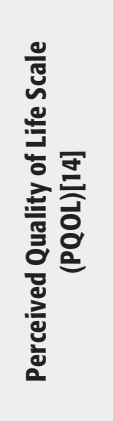 } & \multirow[t]{4}{*}{$\begin{array}{l}\text { No } \\
\text { (generic) }\end{array}$} & \multirow{4}{*}{$\begin{array}{l}19 \text { items total; each graded using 11-point Likert scale } \\
\text { [14] } \\
\text { Subscales: } \\
\text { - Physical health satisfaction ( } 5 \text { items) } \\
\text { - Social health satisfaction (11 items) } \\
\text { - Cognitive health satisfaction ( } 2 \text { items) } \\
1 \text { item related to conversational abilities is not } \\
\text { grouped in any of these subscales } \\
\text { Overall happiness ( } 1 \text { item) may be used to measure } \\
\text { convergent validity. It is not included in any of the } \\
\text { subscales and is administered as a separate question }\end{array}$} & $\begin{array}{l}\text { Overall score and subscale scores may each be } \\
\text { calculated by determining mean or median of } \\
\text { relevant items; score for happiness item is not } \\
\text { part of overall score [14] }\end{array}$ & \multirow[t]{4}{*}{ No } & \multirow[t]{4}{*}{ No } \\
\hline & & & $\begin{array}{l}\text { Minimum score: } 0 \\
\text { Maximum score: } 10\end{array}$ & & \\
\hline & & & \multirow[t]{2}{*}{$\begin{array}{l}\text { Higher the score, greater the satisfaction/ } \\
\text { happiness }\end{array}$} & & \\
\hline & & & & & \\
\hline \multirow{4}{*}{ 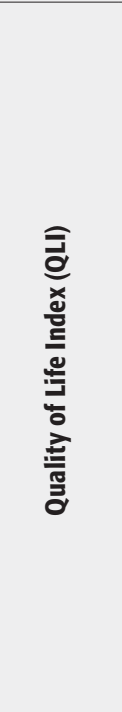 } & \multirow[t]{4}{*}{$\begin{array}{l}\text { Both } \mathrm{SCl} \\
\text { and generic } \\
\text { versions } \\
\text { available }\end{array}$} & \multirow[t]{4}{*}{$\begin{array}{l}4 \text { domains; } 37 / 33 \text { items (SCI/generic version) each } \\
\text { graded using a } 6 \text {-point Likert scale [42]: } \\
\text { - Health and functioning (15/13 items: SCI/generic } \\
\text { version) } \\
\text { - Social and economic ( } 8 / 8 \text { items) } \\
\text { - Psychological/spiritual ( } 7 / 7 \text { items) } \\
\text { - Family (7/5 items) }\end{array}$} & $\begin{array}{l}5 \text { scores may be calculated [42]: } \\
\text { - Total QoL score } \\
\text { - Health and functioning score } \\
\text { - Social and economic score } \\
\text { - Psychological/spiritual score } \\
\text { - Family score }\end{array}$ & \multirow[t]{4}{*}{ Yes $[26,43]$} & \multirow[t]{4}{*}{ No } \\
\hline & & & $\begin{array}{l}\text { Scores calculated by [ }[42] \text { : } \\
\text { 1. Centering scale on } 0 \text { by subtracting } 3.5 \text { from } \\
\text { each item's score } \\
\text { 2. Weight scores with paired importance } \\
\text { responses } \\
\text { 3. Sum all scores (for total QoL score) or } \\
\text { scores for each domain } \\
\text { 4. Calculate mean } \\
\text { 5. Add } 15 \text { to each score to avoid negative } \\
\text { numbers for final score }\end{array}$ & & \\
\hline & & & $\begin{array}{l}\text { Minimum score: } 0 \\
\text { Maximum score: } 30\end{array}$ & & \\
\hline & & & Higher the score, greater the QoL & & \\
\hline
\end{tabular}




\begin{tabular}{|c|c|c|c|c|c|}
\hline $\begin{array}{l}\text { Instru- } \\
\text { ment }\end{array}$ & $\begin{array}{l}\text { Generic } \\
\text { or spine } \\
\text { specific? }\end{array}$ & Scale & Interpretation & $\begin{array}{l}\text { Validated in } \\
\mathrm{SCl} \\
\text { population? }\end{array}$ & $\begin{array}{l}\text { Reliability } \\
\text { tested in SCI } \\
\text { population? }\end{array}$ \\
\hline \multirow{2}{*}{ 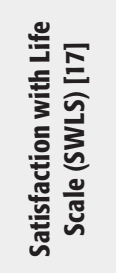 } & \multirow[t]{2}{*}{$\begin{array}{l}\text { No } \\
\text { (generic) }\end{array}$} & \multirow{2}{*}{$\begin{array}{l}5 \text { questions each graded using 7-point Likert scale [17]: } \\
\text { - In most ways my life is close to my ideal } \\
\text { - Conditions of my life are excellent } \\
\text { - I am satisfied with my life } \\
\text { - So far I have gotten important things I want in life } \\
\text { - If I could live my life over, I would change almost } \\
\text { nothing }\end{array}$} & $\begin{array}{l}\text { Minimum score: } 5 \\
\text { Maximum score: } 35\end{array}$ & \multirow[t]{2}{*}{ Yes $[41,44-46]$} & \multirow[t]{2}{*}{ Yes $[41,46]$} \\
\hline & & & Higher the score, greater the life satisfaction & & \\
\hline \multirow{4}{*}{ 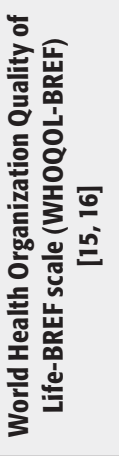 } & \multirow[t]{4}{*}{$\begin{array}{l}\text { No } \\
\text { (generic) }\end{array}$} & \multirow{4}{*}{$\begin{array}{l}4 \text { domains; } 26 \text { items each graded using 5-point Likert } \\
\text { scale }[15,16] \text { : } \\
\text { - Physical health (7 items) } \\
\text { - Psychological health ( } 6 \text { items) } \\
\text { - Social relationships ( } 3 \text { items) } \\
\text { - Environment ( } 8 \text { items) } \\
\text { Items not within a domain: } \\
\text { - Overall QoL ( } 1 \text { item) } \\
\text { - General health (1 item) }\end{array}$} & \multirow{2}{*}{$\begin{array}{l}\text { Scores for each of } 4 \text { domains and } 2 \text { items are } \\
\text { calculated separately [15]: } \\
\text { - Domain scores: mean scores for domain are } \\
\text { multiplied by } 4 \text { (range, } 4-20 \text { ) and this score } \\
\text { may then be transformed to a scale of } 0-100 \\
\text { - Item scores: score is multiplied by } 4 \text { (range, } \\
\text { 4-20) and this score may then be } \\
\text { transformed to a scale of } 0-100\end{array}$} & \multirow[t]{4}{*}{ Yes $[27,47,48]$} & \multirow[t]{4}{*}{ Yes $[27,48]$} \\
\hline & & & & & \\
\hline & & & $\begin{array}{l}\text { Minimum score: } 0 \\
\text { Maximum score: } 100\end{array}$ & & \\
\hline & & & $\begin{array}{l}\text { Higher the score, greater the perception } \\
\text { of QoL }\end{array}$ & & \\
\hline
\end{tabular}

\section{CLINICAL GUIDELINES}

- Little information was found in a search for clinical guidelines recommending measures to evaluate QoL outcomes in patients with SCI.

- The Consortium for Spinal Cord Medicine and the Paralyzed Veterans of America (1999, last reviewed in 2005) recommend that QoL be evaluated using “direct perceptions" of the patient; the Satisfaction with Life Scale (SWLS) was noted as an instrument that can describe subjective QoL[18].

- The Spinal Cord Injury Rehabilitation Evidence project (Canada), which is a collaborative effort among scientists, clinicians, and consumers, recommends the following objective and subjective measures to evaluate QoL in patients with SCI: WHOQOL-BREF, SIP68, and SF-36 (or SF-12) (objective measures); SWLS, LISAT-11, QLI, and QOLP-PD (subjective measures) [19].

- The SCI Consensus Group [20] recommended that QoL be assessed following hospital discharge (from the acute injury) using the SF-36, CHART, LISAT-9, or the Quality of Well-Being Scale (QWB). Before initial discharge, the group recommended using separate measures to assess the component of QoL, including function (Functional Independence Measure), psychological status (Hospital Anxiety and Depression Scale), and pain (Visual Analogue Scale). However, the group noted that the evidence supporting the use of these guidelines is limited and that these recommendations should be reassessed in the future.

\section{DISCUSSION}

- Quality of life is a term used to evaluate individuals' well-being in a wide range of contexts. For patients with SCI, achieving a satisfactory QoL is a primary goal of treatment and rehabilitation. Assessing QoL helps to appraise patients' abilities to adapt and to account for their personal perceptions and values. As such, it should be routinely measured in patients with SCI.

- One proposed scheme divides QoL instruments into two categories: objective QoL and subjective QoL. Objective measures assume all individuals within a culture consider the same domains as important for quality. Furthermore, the degree to which an individual achieves these standards determines the level of quality for that individual. Subjective measures assume that the assessment of quality lies with the individual, and the individual best determines happiness and satisfaction within a framework of personal expectations and accomplishments. 
- The decision to use a particular QoL measure should be based on the study design as well as the predetermined research goals. For example,

- To compare differences in QoL outcome between two groups in a large therapeutic trial, an objective QoL measure would generally be preferred. These have the advantage of assessing externally appraisable QoL characteristics and are usually less time consuming to administer compared with the subjective measures.

- To perform a detailed evaluation through survey of how well-being within a community of patients has been affected by the introduction of a particular intervention, a subjective QoL measure would generally be preferred. These are usually more complex and time consuming, providing an in-depth assessment of QoL within a patient group.

- Wherever possible, use of QoL outcome measures with a defined psychometric profile within the SCI population is preferred.

- Future research should focus on the development and validation of an SCI-specific QoL measurement tool to be used alone or in combination with existing generic measures. It is also likely that different tools may be required when assessing individuals with SCI in the acute, subacute, or chronic phases of their postinjury recovery period.

\section{EDITORIAL STAFF PERSPECTIVE}

The reviewers were unanimous in their approval of this systematic review, and congratulate the authors on identifying this major deficiency in the assessment of spinal cord injury (SCI) outcomes. To our knowledge, this article presents the most comprehensive and systematically organized assessment of outcomes tests used for patients with SCI to date. The findings of this review clearly call into question the continued absence of more specifically developed and validated grading and outcomes tests for the large and ever-growing SCI population.

\section{REFERENCES}

Full reference list can be found in the web appendix at www.aospine.orglebsj.

1. World Health Organization (2001) World Health Organization International Classification of Functioning, Disabilities and Health (ICF). Geneva: World Health Organization.

2. Dijkers MP (2003) Individualization in quality of life measurement: instruments and approaches. Arch Phys Med Rehabil; 84(4 Suppl 2):S3-14.

3. Introduction to the Community Integration Questionnaire. Available at: www.tbims.org/ combi/ciq/. Accessed December 17, 2010.

4. CIQ Syllabus. Available at: www.tbims.org/ combi/ciq/ciqsyl. Accessed December 17, 2010.

5. Craig Hospital Disability Research Instruments: Craig Handicap Assessment and Reporting Technique-"CHART." Available at: www.craighospital.org/research/CHART.asp. Accessed December 16, 2010.

6. Guide for use of the CHART: Craig Handicap Assessment and Reporting Technique. Available at: www.craighospital.org/research/ CHART Manual.pdf. Accessed December 16, 2010.

7. Reintegration to Normal Living (RNL) Index. Available at: www.scireproject.com/outcomemeasures/reintegration-to-normal-living-rnlindex. Accessed December 17, 2010.

8. Reintegration to Normal Living (RNL) Index. Available at: www.medicine.mcgill.ca/ strokengine-assess/pdf/RNLI.pdf. Accessed December 17, 2010.

9. Post MW, de Bruin A, de Witte L, et al (1996) The SIP68: a measure of health-related functional status in rehabilitation medicine. Arch Phys Med Rehabil; 77(5):440-445.

10. Ware J, Jr, Kosinski M, Keller SD (1996) A 12Item Short-Form Health Survey: construction of scales and preliminary tests of reliability and validity. Med Care; 34:220-233.

11. Ware JE Jr, Sherbourne CD (1992) The MOS 36-item short-form health survey (SF-36). I. Conceptual framework and item selection. Med Care; 30:473-483.

12. Fugl-Meyer AR, Bränholm IB, FugI-Meyer KS (1991) Happiness and domain-specific life satisfaction in adult northern Swedes. Clin Rehabil; 5:25-33. 